\title{
An experimental study of the effect of motile bacteria on the fluid displacement in porous media
}

\author{
Boyoung Jeong ${ }^{1}$, Yumeng Zhao ${ }^{1}$, Dong-Hun $\mathrm{Kang}^{1}$, and Sheng Dai ${ }^{1, *}$ \\ ${ }^{1}$ Geosystem Engineering, Georgia Institute of Technology, United States
}

\begin{abstract}
Multiphase flow patterns in porous media largely depend on the properties of the fluids and interfaces such as viscosity, surface tension, and contact angle. Microorganisms in soils change the fluid and interfacial properties, and thus can alter multiphase fluid flow in porous media. This study investigates the impact of motile bacterium Escherichia coli (E. coli) on fluid displacement patterns in a microfluidic chip. The fluid displacement is observed during the saturation and the desaturation processes of the microfluidic chip with and without E.coli suspension. Time-lapse photography results show that the presence of E.coli alters the displacement patterns during the wetting and drying process and changes the residual saturation of the chip. Although studies of the impacts of motility on interfacial properties remain elusive, these results bring the expectation to the manipulation of multiphase transport in porous media and the adaptive control of industrial and environmental flow processes using active particles.
\end{abstract}

\section{Introduction}

Multiphase flow in porous media is crucial in many industrial and environmental processes, such as $\mathrm{CO}_{2}$ sequestration [1], enhanced oil recovery [2], and contaminant transport [3]. The efficiency of these processes is largely governed by the displacement patterns of immiscible fluids in porous media, i.e., viscous finger, capillary finger, and stable displacement [4, 5]. These displacement patterns are inherently governed by fluid viscosity, contact angle, surface tension, and fluid velocity.

Microorganisms living in porous media interact with the surrounding environment and alter their physical properties including permeability, specific surface area, friction, the pore geometry [6-8], and fluid properties like viscosity, surface tension and contact angle [9, 10]. Bacteria are chemically reactive and produce a variety of by-products. In addition, bacteria mechanically interact with the surroundings and change stress distribution within fluids [11]. The fluid-bacteria interactions become overwhelmingly complicated for bacteria with motility $[12,13]$. Fluid rheology affects the movement of motile bacteria, and simultaneously, the motile bacteria induced time-dependent flow and stress filed change can significantly modify the fluid rheological properties [14]. This two-way non-linear coupling between fluids and motile bacteria has profound implications to the abovementioned energy recovery and environmental issues, yet remain poorly understood. The impacts of motile bacteria on the interfacial and contact properties of multiphase fluids in porous media have not been previously investigated [15].
This study presents the first attempt to probe the distinctive difference in two-phase fluid displacement patterns in porous media with and without motile bacteria. Among studied swimming bacteria, Escherichia coli (E.coli) is the most widely investigated at the crossroads of biology, chemistry, physics, and engineering literature. Therefore, we here conduct microfluidics experiments with preliminary results of motile E.coli in altering the displacement patterns and ensued residual fluid saturation in porous media. The results highlight the potential of using active particles to adaptively control multiphase flow in porous media; yet, the impacts of motile bacteria to the interfacial and contact properties are to be furtherly determined.

\section{Experimental study}

\subsection{Setup}

The test setup is shown in Figure 1. All experimental elements except the camera are placed in an incubation box for accurate control of the temperature at $37{ }^{\circ} \mathrm{C}$, which is the optimal temperature for the growth and survival of E.coli. A heating pad and a temperature controller (CN-8PT, Omega) regulate the temperature of the incubation box, and a thermometer located nearby the microfluidic chip records the temperature. A syringe pump (BS-8000, Braintree Scientific Inc) connected with a $3 \mathrm{~mL}$ Syringe pushes the liquid and air into the microfluidic chip with a constant flow rate. A CMOS camera (D5600, Nikon, Japan) with a macro lens (AF-S Micro NIKKOR $60 \mathrm{~mm}$, Nikon, Japan) is right above the

\footnotetext{
* Corresponding author: sheng.dai@ce.gatech.edu
} 
microfluidic chip. An LED panel is placed below the microfluidic chip to provide light for improved imaging.

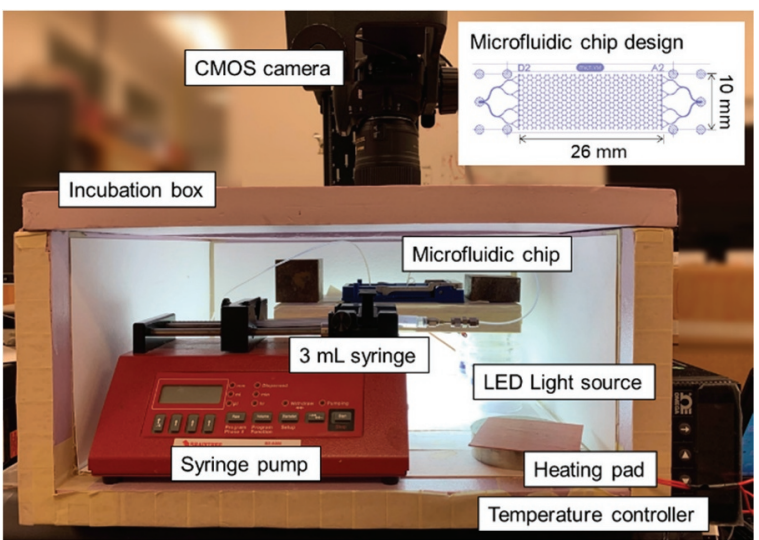

Fig. 1. Test setup for the microfluidic chip experiment. The inset image indicates the design of the microfluidic chip.

The microfluidic chip (illustrated by the inset in Fig. 2 ) is designed and fabricated to succinctly replicate a homogeneous porous medium (Micronit Microtechnologies, Netherlands). The material for the microfluidic chip fabrication is fused silica glass, which is hydrophilic. The operative area of the chip $(10 \times 26 \mathrm{~mm})$ is teeming with uniform circular grains with $800 \mu \mathrm{m}$ in diameter. The center-to-center distance among neighboring grains is $900 \mu \mathrm{m}$, and it brings a pore throat of $100 \mu \mathrm{m}$. The depth of the channel is $40 \mu \mathrm{m}$.

\subsection{Bacteria growth and preparation}

This study selected E.coli ATCC9637 (ATCC, USA) for the motile bacterium strain, which is the model strain of the bacteria motility. E.coli, prevalent in the human intestine, is gram-negative, rod-shaped, and facultative anaerobe. E.coli is $2 \mu \mathrm{m}$ long and $0.5 \mu \mathrm{m}$ wide approximately and has flagella over the cell surface. The flagella rotate and propel the bacterium to help swim back and forth, glide, tumble, and also interact with surrounding fluids $[16,17]$.

Nutrient Broth (NB) medium (ATCC1376) is used for E.coli cultivation, and the Phosphate Buffered Saline (PBS) solution consisting of $0.137 \mathrm{M} \mathrm{NaCl}, 0.0027 \mathrm{M}$ $\mathrm{KCl}, 0.01 \mathrm{M} \mathrm{NaHPO}_{4}$, and $0.0018 \mathrm{M} \mathrm{KH}_{2} \mathrm{PO}_{4}$ is used for cell washing and harvesting, and the experimental suspension. PBS solution is a buffer solution commonly used in the biological study, because of the similar level of osmolality and ion concentration with the human body.

Frozen stock culture is inoculated into $500 \mathrm{~mL}$ of $\mathrm{NB}$ medium and cultivated for 16 hours at $37{ }^{\circ} \mathrm{C}, 180 \mathrm{rpm}$, and $1 \mathrm{~atm}$ conditions in a rotating incubator. The grown E.coli medium is centrifuged ( $2000 \mathrm{rpm}, 10 \mathrm{~min})$ and washed three times with PBS solution and re-diluted into PBS solution. The E.coli concentration of NB medium and PBS solution is measured using a UV spectrometer at $600 \mathrm{~nm}$ and by counting colonies. The E.coli concentration is controlled during the test to secure the reproducibility and reliability, and it lies in $10^{8}-10^{9}$ $\mathrm{CFU} / \mathrm{mL}$

\subsection{Test procedures}

The fluid displacement is observed during the wetting (saturation) and drying (desaturation) processes in the microfluidic chip. Before starting the test, all prepared suspensions and the chip are placed in the incubation box of $37^{\circ} \mathrm{C}$ so that E.coli is the most active in motility.

For the wetting test, a suspension prefilled syringe is connected to the empty microfluidic chip. It should pay heed to minimize the air bubbles in the syringe and connection tube. The LED panel and heating pad are then switched on, and the incubation box is closed to hold on temperature constantly at $37{ }^{\circ} \mathrm{C}$. The suspension is injected with a constant flow rate of $0.1 \mathrm{~mL} / \mathrm{hr}$ into the chip until the fluid percolates the chip and the displacement process becomes a stationary state. The suspension passing through the chip is captured by timelapse photographic images.

The drying test has a similar test procedure as the wetting test. This time, the syringe remains empty, and the chip is fully saturated with suspension. In order to thoroughly saturate the chip without remaining air bubbles, the suspension is pushed into a chip with a high flow rate $(>10 \mathrm{~mL} / \mathrm{hr})$. After the stabilization of temperature, the air is injected into the chips using a syringe pump. The syringe pump consistently pushes the syringe with a flow rate of $0.1 \mathrm{~mL} / \mathrm{hr}$. The test is ended when the air phase reaches the other side of the microfluidic chip.

\subsection{Image processing}

The collected images are processed and analyzed using ImageJ (NIH) and MATLAB (The Mathworks, Inc.). The procedure of image processing is illustrated in Figure 2. The original image (Fig. 2a) obtained from the CMOS camera is segmented into three phases of liquid, air, and grains, as shown in Figure 2b. Each phase is individually highlighted with a different color: blue for liquid, gray for air, and white for grains. Figure $2 \mathrm{c}$ and $2 \mathrm{~d}$ show only the air and the liquid phase, respectively. The saturation of each phase is calculated using the segregated phase area divided by the total pore space.

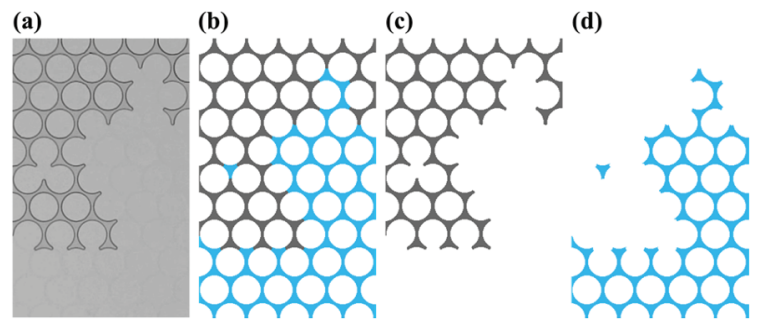

Fig. 2. Procedures of image processing: (a) raw image obtained during the test; (b) segmentation of phases into liquid (blue), air (gray), and grain (white); (c) the air phase; (d) the liquid phase.

\section{Results and Discussion}

Figure 3 shows the processed images of displacement patterns in the saturation tests. The PBS solution with and 
without $E$. coli is injected from the left side of the chip. In both cases, the air phase is quite stably displaced by the suspensions without showing evident fingering phenomena. However, the PBS solution with E.coli displaces the air more effectively than the PBS solution without E.coli. With the presence of E.coli suspension, the chip is more spatially saturated at the end, and the size of entrapped air bubbles is also smaller. The final liquid saturation is $76 \%$ for the PBS solution without E.coli and $87 \%$ for that with E.coli.

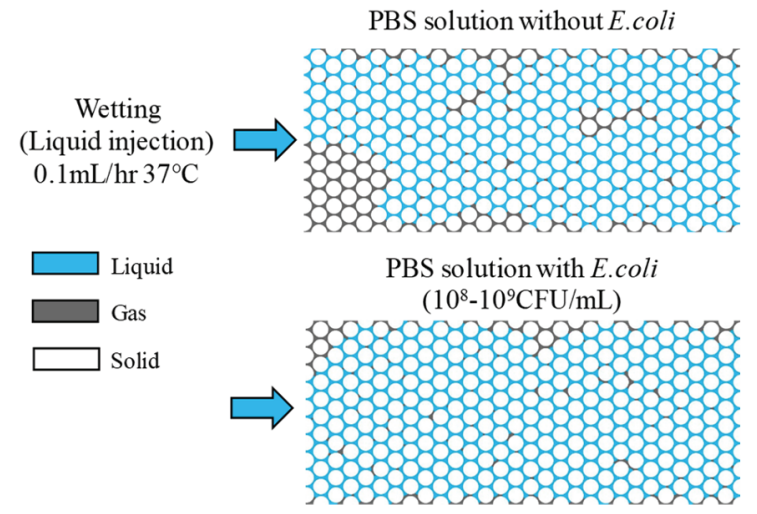

Fig. 3. Displacement patterns after the saturation of microfluidic chip with and without E.coli ATCC9637.

Since the grain surface of the chip is hydrophilic, the injection of E.coli suspension into the air-saturated chip can be considered as imbibition where the wetting fluid displaces the non-wetting fluid. The imbibition in the airwater displacement has not much been investigated. However, the change in displacement patterns can be inferred from the imbibition experiments with other immiscible fluids [4].

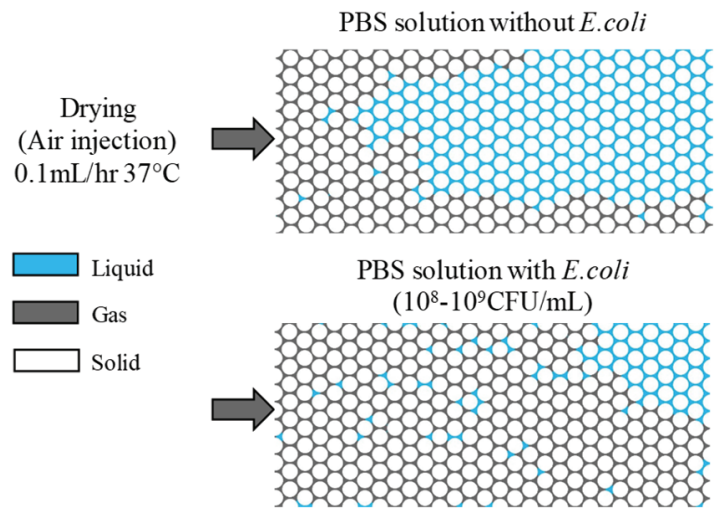

Fig. 4. Displacement patterns after the desaturation of the microfluidic chip with and without E.coli ATCC9637.

Figure 4 presents the distribution patterns at the end of the desaturation tests. The presence of motile E.coli in the solution has more evident impacts in altering the drying process, compared to the wetting process. When air is injected into the liquid saturated chip, the PBS solution without E.coli is displaced with fingering effect, whereas PBS solution with E.coli is replaced with a more stable displacement pattern. And the latter case takes more time for the air to percolate through the chip. The residual liquid saturation in the microfluidic chip is $57 \%$ for the case of PBS solution without E.coli and $18 \%$ for the case of PBS solution with E.coli.

The displacement patterns of two immiscible fluids in porous media (i.e., viscous fingering, capillary fingering, and stable displacement) are governed by two dimensionless numbers: the capillary number $C$ and the viscosity ratio $M$ (Figure 5a). The capillary number $C$ describes the relative magnitude of viscous force to capillary force, and the viscosity number $M$ represents the viscosity ratio of the defending fluid over the invading fluid $[4,5]$. The values of these two dimensionless numbers are directly calculated from fluid viscosity $\mu$, contact angle $\theta$, surface tension $\sigma$, and injection velocity $v_{\text {inj }}$ (Figure 5a). Therefore, at a certain injection rate, the two-phase flow patterns in porous media are innately governed by $\mu, \theta$, and $\sigma$, which can be altered due to the presence of motile $E$. coli.
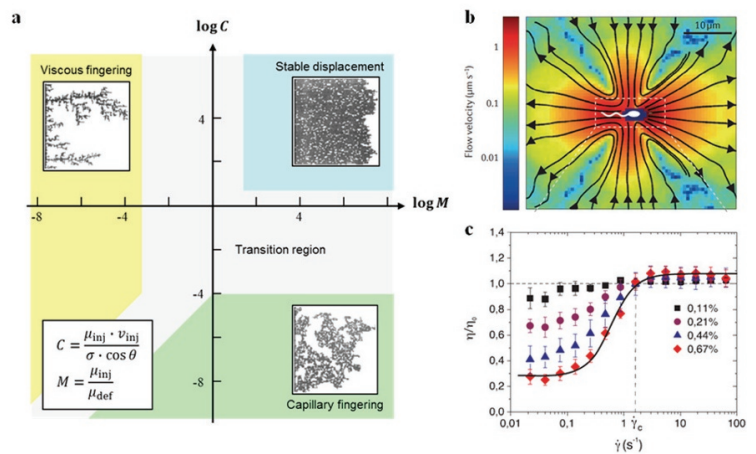

Fig. 5. Impacts of motile E. coli on two-phase flow in porous media. (a) The displacement patterns of two-phase flow in porous media are governed by dimensionless viscous $M$ and capillary $C$ numbers [5]. (b) The presence of motile E. coli alters the stress distribution in fluid [11]. (c) E. coli reduces fluid viscosity at low shear rates [9].

The reduction of effective fluid viscosity at low shear rate due to motile $E$. coli has been experimentally observed [9] and also analytically and theoretically studied $[18,19]$. However, no previous studies have investigated the impact of motile E. coli on contact angle and surface tension, which are in need of in-depth studies to better understand the role of motile E. coli in multiphase flow in porous media.

The impacts of motile bacteria to the fluid and interfacial properties are strongly influenced by the bacteria concentration, motility, and motion characteristics, as well as the rheological properties of the host fluids. Newtonian fluids with motile $E$. coli present strong shear rate dependent and non-Newtonian behaviors $[11,20]$ that challenge the traditional definitions of $C$ and $M$ numbers. And thus, a new theoretical framework of multiphase fluids with active suspension in porous media is in great need. Nevertheless, this study presents evident changes that motile E. coli may introduce in two-phase flow in a microfluidic chip. 


\section{Conclusions}

This study investigates the displacement patterns of two immiscible fluids (i.e., air and PBS solution) through a microfluidic chip, considering the impacts of motile $E$. coli. The results demonstrate that the presence of E.coli assertively alters the displacement patterns of both the saturation and desaturation processes in the microfluidic chip. With $10^{8}-10^{9} \mathrm{CFU} / \mathrm{mL}$ E. coli, the saturation rate increases from $76 \%$ to $87 \%$ at a fluid injection rate of 0.1 $\mathrm{mL} / \mathrm{hr}$. During the drying tests, $82 \%$ of the PBS solution with $10^{8}-10^{9} \mathrm{CFU} / \mathrm{mL}$ E. coli can be drained by injected air at a rate of $0.1 \mathrm{~mL} / \mathrm{hr}$, while only $43 \%$ of PBS solution without E. coli can be drained at an identical air injection condition. The fluid rheological and interfacial properties are no longer constant values due to the collective motion of $E$. coli, and thus challenge the quantification of the microfluidic chip results presented here. However, it is evidenced that motile $E$. coli can significantly alter the displacement patterns and the efficiency of multiphase transport in porous media. These results help to be more informed about using active suspensions to manipulate multiphase transport in porous media and offering the potential to adaptively control flow in many industrial and environmental processes.

This material is based upon work supported by the National Science Foundation (EEC-1449501 and CMMI-1943722). Any opinions, findings and conclusions, or recommendations expressed in this material are those of the authors and do not necessarily reflect those of the NSF.

\section{References}

1. Kang, Q., et al., Pore scale modeling of reactive transport involved in geologic CO 2 sequestration. Transport in porous media, 2010. 82(1): p. 197-213.

2. Zhao, X., M.J. Blunt, and J. Yao, Pore-scale modeling: Effects of wettability on waterflood oil recovery. Journal of Petroleum Science and Engineering, 2010. 71(3-4): p. 169-178.

3. Held, R.J. and M.A. Celia, Pore-scale modeling and upscaling of nonaqueous phase liquid mass transfer. Water Resources Research, 2001. 37(3): p. 539-549.

4. Lenormand, R., Liquids in porous media. Journal of Physics: Condensed Matter, 1990. 2(S): p. SA79.

5. Lenormand, R., E. Touboul, and C. Zarcone, Numerical models and experiments on immiscible displacements in porous media. Journal of fluid mechanics, 1988. 189: p. 165-187.

6. Cunningham, A.B., et al., Influence of biofilm accumulation on porous media hydrodynamics. Environmental science \& technology, 1991. 25(7): p. 1305-1311.

7. Deng, W., et al., Effect of Permeable Biofilm on Micro-And Macro-Scale Flow and Transport in Bioclogged Pores. Environmental science \& technology, 2013. 47(19): p. 11092-11098.

8. Kim, J.-W., H. Choi, and Y.A. Pachepsky, Biofilm morphology as related to the porous media clogging. Water Research, 2010. 44(4): p. 1193-1201.
9. López, H.M., et al., Turning bacteria suspensions into superfluids. Physical review letters, 2015. 115(2): p. 028301.

10. Bakhshi, N., S. Soleimanian-Zad, and M. SheikhZeinoddin, Dynamic surface tension measurement for the screening of biosurfactants produced by Lactobacillus plantarum subsp. plantarum PTCC 1896. Enzyme and microbial technology, 2017. 101: p. 1-8.

11. Son, K., D.R. Brumley, and R. Stocker, Live from under the lens: exploring microbial motility with dynamic imaging and microfluidics. Nature Reviews Microbiology, 2015. 13(12): p. 761.

12. Lauga, E. and T.R. Powers, The hydrodynamics of swimming microorganisms. Reports on Progress in Physics, 2009. 72(9): p. 096601.

13. Giomi, L., T.B. Liverpool, and M.C. Marchetti, Sheared active fluids: Thickening, thinning, and vanishing viscosity. Physical Review E, 2010. 81(5): p. 051908.

14. Patteson, A.E., A. Gopinath, and P.E. Arratia, Active colloids in complex fluids. Current Opinion in Colloid \& Interface Science, 2016. 21: p. 86-96.

15. Zhao, Y., B. Jeong, and S. Dai. Impacts of motile Escherichia coli on surface tension. in ICEGT2020. 2020. La Jolla.

16. Berg, H.C., E. coli in Motion. 2008: Springer Science $\&$ Business Media.

17. Purcell, E.M., Life at low Reynolds number. American journal of physics, 1977. 45(1): p. 3-11.

18. Haines, B.M., et al., Three-dimensional model for the effective viscosity of bacterial suspensions. Physical Review E, 2009. 80(4): p. 041922.

19. Saintillan, D., The dilute rheology of swimming suspensions: A simple kinetic model. Experimental Mechanics, 2010. 50(9): p. 1275-1281.

20. Alonso-Matilla, R., B. Ezhilan, and D. Saintillan, Microfluidic rheology of active particle suspensions: Kinetic theory. Biomicrofluidics, 2016. 10(4): p. 043505 . 